\title{
Diabetic neuropathy: promises and disappointments from benchside to bedside
}

\section{Abbreviations}

WHO: world health organization; MG: methyl glyoxal; DRG: dorsal root ganglia; BNB: blood nerve barrier; BBB: blood brain barrier

\section{Editorial}

Diabetic neuropathy is the most common complication of diabetes and according to World Health Organization (WHO) almost $50 \%$ of diabetes affected patients have same degree of neuropathy ${ }^{1}$. In terms of health care costs, diabetic peripheral neuropathy has a high postdiagnosis economic impact being associated with a $20 \%$ increase in the number of patients visiting hospitals and a $46 \%$ increase in the number of visits to hospitals compared to the pre-diagnosis situation. ${ }^{2}$ As diabetic neuropathy predisposes to the diabetic foot disease, it should be also considered as one of the major causes determining the lower limb amputation. In fact, diabetic neuropathy increases by 7-fold the risk of foot ulceration. ${ }^{3}$ Diabetic neuropathy is associated with nerve damage and should be considered as an important factor contributing to disability, sexual dysfunction, urinary tract infections, digestive problems, social isolation, and depression. ${ }^{4}$ Last by not least, diabetes increases by 2-4 fold the risk of stroke. ${ }^{5}$ Therefore, social costs imply beside the effective health care costs for diabetes treatment, an additional budget for the social security disability, impotence treatment, depression treatment etc. Considering the variety of aspects, from health to economical ones, it is imperative to find new solutions (e.g. molecules/markers) for early diagnosis of diabetes/diabetic neuropathy and a concerted effort of the public and private funding should be focused to the benchside.

Classical tests for diabetes diagnosis, such as glycohemoglobin test, fasting plasma glucose test and oral glucose tolerance test, are often done in late diabetes stages when diabetic neuropathy is already installed. A new threat in early diabetic neuropathy diagnosis is represented by corneal confocal microscopy, but its correlation with intra epidermal nerve fiber density, quantitative sensory testing or autonomic function testing is still controversial ${ }^{6}$. It is necessary to revise the early diabetes diagnosis in order to improve the prognostic for diabetic neuropathy and other complications.

There is growing evidence that methylglyoxal $(\mathrm{MG})$ is a key player in diabetic neuropathy and its plasma level monitoring has clinical relevance. Indeed, a recent study discriminates painful and non-painful diabetic neuropathy in diabetic patients based on the MG plasma levels $(>600 \mathrm{nM})$. Interestingly, the same MG changes were identified in streptozotocin-induced and genetic mouse models of diabetes. At the molecular level, MG depolarizes mouse dorsal root ganglia (DRG) neurons, ${ }^{7}$ exerts a dual effect on the viability, excitability, and neurite outgrowth of these neurons, ${ }^{8}$ induces post-translational modifications of the voltage-gated sodium channel Nav1.8 and determines excitability changes correlated with the neuronal somasize. ${ }^{7} \mathrm{MG}$ glycation mediates peripheral micro vascular complications (e.g. nephropathy, retinopathy, and neuropathy). Furthermore,
Volume I Issue 3 - 2014

\author{
Beatrice Mihaela Radu, ${ }^{1,2}$ Daniel Dumitru \\ Banciu, ${ }^{2}$ Adela Banciu, ${ }^{2}$ Mihai Radu ${ }^{1,3}$ \\ 'Department of Neurological and Movement Sciences, \\ University of Verona, Italy \\ ${ }^{2}$ Department of Anatomy, University of Bucharest, Romania \\ ${ }^{3}$ Department of Life and Environmental Physics, 'Horia Hulubei' \\ National Institute of Physics and Nuclear Engineering, Romania
}

Correspondence: Beatrice Mihaela Radu, Department of Neurological and Movement Sciences, University of Verona, Strada Le Grazie 8,Verona, Italy, Tel 00-39-0458027670, Email beatricemihaela.radu@univr.it

Received: July 17, 2014 | Published: July 18, 2014

experimental diabetic nephropathy has been shown to be prevented by genomic manipulation of the glyoxalase 1 expression that regulates the MG glycation. ${ }^{9}$ Therefore, MG is a good example for a candidate molecule to be included in current diagnosis screening tests.

The traditional concept that painful neuropathy is a complication of diabetes tends to change into a channelopathies-based theory. ${ }^{10}$ Extensive literature is dedicated to down/up-regulated of various ion channels expressed in DRG sensory neurons in relationship with experimental or clinical diabetic neuropathy, such as Nav1.8, Nav1.7, TRPV1, Cav 3.2 T-type calcium channel, ASIC1-ASIC3, etc. ${ }^{7,10-13}$ However, yet clinical practice is limited by the unknown contribution of channelopathies vs vasculitis affecting the vasa nervorum in diabetic neuropathy.

Micro vascular dysfunctions in diabetes are often called 'diabetic complications' but them seem to be rather a cause than a consequence. To be more precise, micro vascular dysfunctions in diabetes occur both at the level of blood-nerve barrier (BNB; being correlated to diabetic neuropathy) and blood brain barrier (BBB; being correlated to stroke). Blood markers indicating $\mathrm{BNB}$ and $\mathrm{BBB}$ leakage ${ }^{14-16}$ could also represent a promising screening test and constitute a good approach for early diagnosis of diabetic neuropathy and stroke risk associated to diabetes.

We strongly believe that already known molecules from basic diabetes research, that might have a great potential for early diabetic neuropathy diagnosis, should be considered in the clinical practice for the screening of 'healthy' population, especially when there is a genetic predisposal.

\section{Acknowledgements}

DD Banciu is currently supported by the Sectorial Operational Programme Human Resources Development (SOPHRD), financed by the European Social Fund and the Romanian Government under the contract number POSDRU/159/1.5/S/141531. 


\section{Conflicts of interest}

The authors declare there are no conflicts of interest related to the article.

\section{References}

1. http://www.who.int/mediacentre/factsheets/fs138/en/

2. Mehra M, Merchant S, Gupta S, et al. Diabetic periphera neuropathy:resource utilization and burden of illness. J Med Econ 17:1-9.

3. Khanolkar MP, Bain SC, Stephens JW. The diabetic foot. QJM 2014;101(9):685-695.

4. http://www.mayoclinic.org/diseases-conditions/diabetic-neuropathy/ basics/complications/con-20033336.

5. National Stroke Association.

6. Malik RA. Which test for diagnosing early human diabetic neuropathy? Diabetes. 2014;63(7):2206-2208

7. Bierhaus A, Fleming T, Stoyanov S, et al. Methylglyoxal modification of Nav1.8 facilitates nociceptive neuron firing and causes hyperalgesia in diabetic neuropathy. Nat Med. 2012;18(6):926-933.

8. Radu BM, Dumitrescu DI, Mustaciosu CC, et al. Dual effect of methylglyoxal on the intracellular $\mathrm{Ca} 2+$ signaling and neurite outgrowth in mouse sensory neurons. Cell Mol Neurobiol. 2012;32(6):1047-1057.

9. Rabbani N, Thornalley PJ. The critical role of methylglyoxal and glyoxalase 1 in diabetic nephropathy. Diabetes. 2014;63(1):50-52.
10. Hoeijmakers JG, Faber CG, Merkies IS, et al. Channelopathies, painful neuropathy, and diabetes:which way does the causal arrow point? Trends Mol Med. 2014;20(10):544-550.

11. Radu BM, Iancu AD, Dumitrescu DI, et al. TRPV1 properties in thoracic dorsal root ganglia neurons are modulated by intraperitoneal capsaicin administration in the late phase of type- 1 autoimmune diabetes. Cell Mol Neurobiol. 2013;33(2):187-196.

12. Khomula EV, Viatchenko-Karpinski VY, Borisyuk AL, et al. Specific functioning of Cav3.2 T-type calcium and TRPV1 channels under different types of STZ-diabetic neuropathy. Biochim Biophys Acta. 2013;1832(5):636-649.

13. Radu BM, Dumitrescu DI, Marin A, et al. Advanced type 1 diabetes is associated with ASIC alterations in mouse lower thoracic dorsal root ganglia neurons. Cell Biochem Biophys. 2014;68(1):9-23.

14. Shimizu F, Sano Y, Haruki H, et al. Advanced glycation end-products induce basement membrane hypertrophy in endoneurial micro vessels and disrupt the blood-nerve barrier by stimulating the release of TGF- $\beta$ and vascular endothelial growth factor (VEGF) by pericytes. Diabetologia. 2011;54(6):1517-1526.

15. Serlin Y, Levy J, Shalev H. Vascular pathology and blood-brain barrier disruption in cognitive and psychiatric complications of type 2 diabetes mellitus. Cardiovasc Psychiatry Neurol. 2011;2011:609202.

16. Radu BM, Bramanti P, Osculati F, et al. Neurovascular unit in chronic pain. Mediators Inflamm. 2013;2013:648268. 